\title{
DETERMINANTES PARA UTILIZAÇÃO DE HEDGE ACCOUTING: UMA ESCOLHA CONTÁBIL
}

\section{DRIVERS FOR THE USE OF HEDGE ACCOUNTING: AN ACCOUNTING CHOICE}

\section{DETERMINANTES PARA UTILIZACIÓN DE HEDGE ACCOUTING: UNA ELECCIÓN CONTABLE}

\author{
FERNANDO CAIO GALDI \\ Professor Associado da Fucape Business School e Coordenador de Pesquisas Fucape \\ Business School, Doutor em Ciências Contábeis pela USP \\ fernando.galdi@fucape.br
}

LUIZ FERNANDO GRAMA GUERRA

Mestre em Contabilidade pela Fucape Business School

Iguerra@samarco.com

\section{RESUMO}

Este estudo investiga a sistemática da escolha contábil, no meio corporativo, referente à contratação e à qualificação, ou não, de operações com derivativos para aplicação da contabilidade de hedge (Hedge Accounting), conforme as normas do Fasb (SFAS 133). Dentro dessa abordagem, foram utilizadas variáveis relacionadas aos incentivos/benefícios que as empresas podem ter ao classificarem uma transação dentro dos requisitos necessários para aplicação da contabilidade de operações de hedge (Hedge Accounting). As empresas avaliadas são aquelas listadas na NYSE nos setores de Mineração, Siderurgia/Metalurgia e Papel/Celulose. Os dados utilizados foram obtidos por meio da base de dados Economatica, websites das firmas selecionadas e da análise das demonstrações financeiras publicadas nos relatórios anuais, em dólares norte-americanos, de cada uma das selecionadas firmas, referentes ao ano de 
2006. As evidências apontam para uma relação positiva e significante entre a dívida de longo prazo e a aplicação da contabilidade de hedge, o que é consistente com a teoria e corrobora com a relação esperada.

Palavras-chave: Hedge Accounting, Derivativos, Transação.

\section{ABSTRACT}

This study investigates the incentives related to accounting choices regarding the qualification of derivatives in the corporate context for hedge accounting, or not, according to SFAS 133. We consider variables related to incentives/benefits that firms could have by qualifying an operation for hedge accounting. The assessed companies were from the Mining, Industrial Metals and Cellulose/Paper sectors listed on the NYSE. The data used in this study was obtained from the Economatica database and firms' websites for the year 2006. Evidences indicates a positive and significant relation between a firm's long term debt and hedge accounting qualification, which is consistent with theory and confirms the expected relation.

Keywords: Hedge Accounting, Derivatives, Transaction.

\section{RESUMEN}

Este estudio investiga la sistemática de la elección contable, en el medio corporativo, referente a la contratación y a la calificación, o no, de operaciones con derivativos para aplicación de la contabilidad de hedge (Hedge Accounting), conforme a las normas del Fasb (SFAS 133). Dentro de ese abordaje, fueron utilizadas variables relacionadas a los incentivos/beneficios que las empresas pueden tener al clasificar una transacción dentro de los requisitos necesarios para aplicación de la contabilidad de operaciones de hedge (Hedge Accounting). Las empresas evaluadas son aquellas listadas en la NYSE en los sectores de Minería, Siderurgia/Metalurgia y Papel/Celulosa. Los datos utilizados fueron obtenidos por medio de la base de datos Economatica, websites de las firmas seleccionadas y del análisis de las demostraciones financieras publicadas en los informes anuales, en dólares norteamericanos, de cada una de las seleccionadas firmas, referentes al año 2006. Las evidencias apuntan hacia una relación positiva y significante entre la deuda de largo plazo y la aplicación de la contabilidad de hedge, lo que es consistente con la teoría y corrobora con la relación esperada.

Palabras clave: Hedge Accounting, Derivativos, Transacción. 


\section{INTRODUÇÃO}

A necessidade de fornecimento de informações para apoiar decisões de investimento é essencial, porém, o predomínio da falta de padronização na elaboração e na evidenciação das demonstrações financeiras ainda pode ser considerado como um obstáculo. A crescente complexidade do meio corporativo gerou maior demanda por uniformização do tratamento contábil por parte de companhias com a intenção de se financiar por intermédio de abertura de capital. O resultado da pressão ocasionada pela mencionada demanda foi a criação do Financial Accounting Standards Board (FASB), em 1973, que possibilitou a formulação de uma estrutura conceitual contábil mais sólida.

Segundo as normas do FASB conhecidas como United States Generally Accepted Accounting Practices (USGAAP), por meio de um de seus pronunciamentos iniciais, o SFAC n. ${ }^{\circ} 2$ - Qualitative Characteristics of Accounting Information, sugere-se que a informação contábil contenha características qualitativas consideradas de grande importância para a tomada de decisão pelos gestores de empresas e também pelos usuários externos, como compreensibilidade, relevância, confiabilidade e comparabilidade. Obviamente, o equilíbrio entre o alcance de tais características nas informações liberadas e o custo e o benefício das mesmas deve ser avaliado.

Os avanços do conhecimento e do ambiente empresarial moderno modificam a percepção de usuários internos e externos, levando-os a necessitar de novas informações contábeis e/ou sua apresentação de formas diferentes. É o caso do grande enfoque atual na chamada Governança Corporativa, principalmente no que se refere ao grau de disclosure ou evidenciação, que tende a evoluir e a se consolidar de uma vez por todas no meio empresarial, e isso é refletido na preocupação crescente das firmas em agir conforme o melhor interesse dos acionistas.

O uso de derivativos vem se tornando cada vez mais comum, devido à sua grande eficácia. Essa "popularização" comprova a necessidade de maximização do valor da empresa para os detentores de ações. Tais instrumentos podem também ser utilizados com fins especulativos frente ao risco de mercado com o intuito de aumentar a alavancagem financeira, porém, neste caso, não devem ser designados como instrumentos de proteção ou de hedge.

Conforme pesquisa realizada por Saito e Schiozer (2005, p. 97-107), as evidências sugerem que, atualmente, as firmas se utilizem de derivativos com o principal intuito de gerenciar riscos por meio da diminuição da volatilidade do resultado. Concluem também que existe maior preocupação com aspectos institucionais e legais (tributação e tratamento contábil) do que com econômicos. Ainda segundo o estudo mencionado, as principais e potenciais fontes de volatilidade são a variação cambial, as taxas de juros e os preços de commodities, não necessariamente nesta ordem. DeMarzo e Duffie (1995, p. 743-771) cor- 


\section{repec}

roboram com a afirmação anterior e alegam que um dos motivos é o acesso de gestores à informação acurada sobre a exposição das mencionadas fontes nas companhias e, assim, consequentemente, devido a esse nível de assimetria natural, tais gestores se encontrariam em melhor posição para evitar os mencionados riscos por meio de operações de hedge.

Para melhor contextualização, segundo Lima e Lopes (2001, p. 27), os chamados derivativos possuem características que os diferem dos demais instrumentos financeiros, que são a alta alavancagem, devido à sua possibilidade de gerar grandes prejuízos; a grande velocidade nas transações, por conta do avanço da tecnologia atual, possibilitando assim a efetivação de operações em questão de segundos em terminais eletrônicos; e a enorme complexidade da estrutura dos produtos, fazendo-se necessário seu adequado entendimento, principalmente, na área de controladoria das empresas, uma vez que as informações podem se tornar obsoletas rapidamente. Os principais tipos de derivativos negociados são os Contratos a Termo ou Forward que, normalmente, são contratados entre empresas e bancos (mercado balcão) e possuem contratos não-padronizados com ajuste na liquidação; os Contratos Futuros, negociados em bolsas de valores com ajustes diários; as Opções de Compra e Venda, também conhecidas como Call e Put, respectivamente, que envolvem direitos de compra e venda de contratos no futuro, pelos quais se paga um prêmio; e as Operações de SWAP que, conforme a própria tradução da palavra para o Português, refere-se a contratos de troca do fluxo financeiro de variáveis (ativo ou passivo) relacionadas a um montante principal, sem que este último seja permutado.

Em junho de 1998, o FASB emitiu o pronunciamento SFAS 133 - Standard Accounting for Derivative Instruments and Hedging Activities, com o intuito de regulamentar a contabilização de derivativos, e que, posteriormente, foi complementado pelo pronunciamento SFAS 138 - Accounting for Certain Derivative Instruments and Certain Hedging Activities An Amendment of FASB Statement $N .^{\circ} 133$, uma vez que o primeiro recebeu muitas críticas devido à sua dificuldade de adaptação/implementação (o início de sua data de vigência foi postergada para junho de 2000, pelo SFAS 137 - Accounting for Derivative Instruments and Hedging Activities - Deferral of the Effective Date of FASB Statement N. ${ }^{\circ} 133$ ).

O uso de derivativos relacionados a moedas e a taxas de juros está associado com o alto valor das empresas em diversos países. Operações como estas podem criar um nível de volatilidade adicional nas contas de resultado, causando impactos negativos, diante da visão de agências de classificação de risco, investidores e instituições de crédito no que se refere ao desígnio de especulação ou proteção. Assim, muitas firmas tentam evitar tal efeito por meio da adoção de padrões contábeis internacionais, cujos resultados são testados e consolidados, apesar de serem também criticados. Conforme Smith e Stulz (1985), a redução na volatilidade de lucros e fluxos de caixa pode fazer com que o valor das empresas aumente na presença de imperfeições de mercado, como, por exemplo, é o caso do chamado Financial Distress, situação em que a firma tem dificuldade de honrar seus compromissos 
financeiros com seus credores, podendo incorrer em custos extras como financiamentos mais caros. Ainda conforme Smith e Stulz (1985), a mitigação da oscilação nos resultados também tem o papel de emitir sinais ao mercado de melhora da qualidade de gerenciamento das empresas. É com este intuito (proteção) que operações de hedge, no caso específico, podem ser classificadas como Hedge Accounting. Segundo Lopes e Santos (2003, p. 18), um dos motivos do surgimento do modelo Hedge Accounting foi a necessidade de se minimizar o efeito causado pelas diferenças de mensuração entre o item protegido e o instrumento de hedge, respeitando o princípio contábil da confrontação das receitas e das despesas, uma vez que tais diferenças resultavam no reconhecimento em Resultado dos ganhos e perdas compensatórios em períodos diferentes.

Para melhor esclarecimento, segundo Carvalho (2002, p. 84), as mencionadas diferenças surgem devido às decisões sobre o reconhecimento contábil e a mensuração de ativos e passivos que são tomadas individualmente, sem considerar o inter-relacionamento com outros ativos e passivos. Desta maneira, alguns ativos e passivos são reconhecidos na Demonstração de Resultados e outros, como, por exemplo, um compromisso firme não o é. No que se refere à mensuração, acontece o mesmo, isto é, enquanto alguns são reconhecidos pelo custo histórico, outros são medidos pelo valor corrente ou pelo menor valor entre custo e mercado.

Devido à grande complexidade estabelecida no pronunciamento SFAS 133, no que se refere à forma de enquadramento e êxito do chamado Teste de Efetividade, que nada mais é do que o cumprimento de requisitos preestabelecidos, muitas firmas, antes de contratarem as mencionadas operações, estabelecem um período de tempo considerável para o alinhamento de sua política de risco e adequação de procedimentos. Os custos envolvidos em operações como essas são altos, consequentemente, a avaliação dos mesmos e de seus benefícios, de forma a encontrar um nível ótimo para maximizar o valor da companhia, é essencial, assim como a constatação de que variáveis podem influenciar a mencionada escolha contábil.

Diante do contexto apresentado, objetiva-se efetuar, por meio da identificação do poder explicativo de informações contábeis relacionadas a proxies predeterminadas, inferências sobre as decisões para utilização de operações de derivativos, como Hedge Accounting.

A hipótese a ser trabalhada por meio do desenvolvimento de um modelo de regressão logit auxiliará no esclarecimento da seguinte questão-problema: os fatores "tamanho" e "dívida" influenciam positivamente a escolha de companhias no que se refere à aplicação de contabilidade de hedge (Hedge Accounting) em suas operações?

A refutação ou a confirmação das hipóteses darão base para a resposta da questão problema; $h_{0}$ : a opção pela aplicação da contabilidade de operações de hedge não é influenciada positivamente pelo tamanho e dívida das empresas; e h1: a opção pela aplicação da contabilidade de operações de hedge é influenciada positivamente pelo tamanho e dívida das empresas. 
A amostra é composta por empresas listadas na Bolsa de Valores de Nova lorque (NYSE) do setor de Mineração, Siderurgia e Metalurgia e Papel e Celulose, cujos dados foram delimitados pelo ano de 2006. Apesar de a amostra compreender apenas os mencionados setores, a intenção foi encontrar resultados consistentes que pudessem permitir a identificação de determinantes que exercem influência sobre a referida escolha contábil por meio de inferências. A seleção de setores econômicos específicos se fez devido à sua relação direta com commodities, cujos preços têm oscilação constante e que, por sua vez, se refletem nas variáveis contábeis das firmas pesquisadas e na sua escolha pela aplicação da contabilidade de operações hedge (Hedge Accounting). É importante ressaltar que, por se tratar de um estudo relacionado a uma escolha contábil, a delimitação pelo ano de 2006 é suficiente para gerar resultados relevantes, de forma a possibilitar melhor compreensão e discussões mais aprofundadas sobre o assunto por parte de empresas e agentes econômico-financeiros. Isso contribui para a otimização da eficiência de tais operações no mercado financeiro, uma vez que a convergência para normas contábeis internacionais é um fato real e a evolução/expansão do entendimento do referido tema se faz necessária.

Como já mencionado, as pesquisas de Saito e Schiozer (2005) e Fenn, Post e Sharpe (1996) forneceram base para o presente trabalho, uma vez que sugerem a existência de correlação entre o tamanho e a dívida das companhias e o uso de derivativos, possibilitando assim a referência às variáveis selecionadas e enfatizando a possibilidade de obtenção de incentivos/benefícios econômicos provindos do modelo de Hedge Accounting.

\section{REVISÃO DA LITERATURA}

\subsection{Hedge Accounting - Características}

O SFAS 133 define conceitos e estabelece padrões de contabilização e emissão de relatórios para instrumentos de derivativos e atividades de hedge, para que, dessa forma, haja um procedimento assertivo, que o seu tratamento seja padronizado e que sejam reconhecidas em balanço patrimonial, uma vez que estabelecem direitos e obrigações para as partes envolvidas. Um ponto importante a se observar é que tais direitos e obrigações são estimados, pois ainda não ocorreram e, dessa maneira, contabilizar-se-á um valor futuro, o que pode ser percebido como um avanço da Contabilidade, mas, ao mesmo tempo, devido ao fator subjetividade, também como uma necessidade de refinamento por meio de estudos empíricos mais aprofundados.

Para que um instrumento possa ser considerado como um derivativo, segundo o FASB, ele deve possuir uma ou mais variáveis às quais se refere (variáveis base) e também um ou mais valores nocionais (especificados em contrato) ou provisões de pagamentos ou ambos; b) possuir um pequeno ou nulo investimento inicial; c) possuir termos que requerem ou permi- 
tem a liquidação da posição líquida financeira, isto é, através de compensação, ou entrega de um ativo com condições de liquidez financeira semelhantes. O mencionado pronunciamento requer que a empresa reconheça todos os derivativos como ativo ou passivo em seu Balanço Patrimonial e os meça, juntamente com seus respectivos instrumentos, ao seu fair value, que é considerado como a mais relevante medida para instrumentos financeiros e a única para derivativo. $O$ importante é manter a consistência do método de mensuração selecionado ao longo do período de vigência, conforme a estratégia de gerenciamento de risco estabelecida pela empresa, e proporcionar sua evidenciação em notas explicativas.

Segundo o SFAS 133, os ganhos e perdas referentes a instrumentos de hedge ou variações de seu fair value devem ser contabilizados conforme sua pretensão de uso e classificação. Lima e Lopes (2001, p. 32) interpretam a classificação estabelecida pelo SFAS 133 como:

1. Hedge de uma exposição a uma mudança no fair value de um ativo ou passivo já reconhecido ou de um compromisso ainda não reconhecido (Fair Value Hedge);

2. Hedge de uma exposição a fluxos de caixa variáveis de uma transação futura projetada (Cash Flow Hedge);

3. Hedge de uma exposição a uma moeda estrangeira de um investimento em uma operação internacional, um compromisso ainda não reconhecido, um título destinado a operação de trading da instituição ou uma transação esperada em moeda estrangeira;

4. Instrumentos não designados como instrumentos de hedge - estes são aqueles que a instituição adquiriu com o propósito de obter resultados positivos com as flutuações de preços e taxas.

A norma SFAS 133 não especifica uma metodologia em particular, deixando à escoIha das firmas o método de avaliação de efetividade que acreditarem ser o mais apropriado. Porém, ela exige que o método selecionado seja utilizado de forma continuada e que outras operações de hedge sejam avaliadas de maneira similar. Os procedimentos adotados devem possuir base justificável para execução.

\subsection{Contabilização}

Com relação à contabilização, em operações de Fair Value Hedge, as variações são reconhecidas no mesmo período que os correspondentes itens de hedge, isto é, basicamente a metodologia estabelecida para a contabilidade de operações de hedge (Hedge Accounting) assegura que a compensação de ganhos e perdas advindas de instrumentos de hedge afetem o Resultado ao mesmo tempo que os ganhos e perdas dos itens de hedge, resultando na mitigação de sua volatilidade, uma vez que os números gerados serão menores (o procedimento assegura que apenas a porção não-efetiva 
seja reconhecida). Isso reflete a alta correlação entre a mudança de fair value do instrumento de hedge e a respectiva mudança no valor do item de hedge.

Já os ganhos e perdas provindos de operações de cash flow hedge, se considerados efetivos ao obter sucesso no método de cálculo já citado e conhecido como Teste de Efetividade adotado pelas companhias, devem ser diferidos em subconta de Patrimônio Líquido denominada como Other Comprehensive Income $(\mathrm{OCI})$ e, quando o item de hedge (transação futura) vier a ocorrer, afetando assim o Resultado, os valores acumulados em $\mathrm{OCl}$ deverão ser reclassificados para aquela mesma conta, de forma a se compensarem com os ganhos e perdas de tais transações (variações dos itens de hedge). O efeito final se assemelha ao gerado em operações de fair value hedge, isto é, as variações que se referem à porção efetiva se anulam e somente a parte não-efetiva é que tem efeito de registro em Resultado.

No caso de operações de Hedge Accounting onde há exposição a moeda estrangeira, o tratamento deve ser efetuado conforme a natureza da operação, isto é, Fair Value ou Cash Flow Hedge.

Mudanças no fair value de derivativos não-classificados como Hedge Accounting são registradas imediatamente em Resultado, isto é, no mesmo período em que ocorre, causando assim grande oscilação naquela conta e, no mínimo, desconforto para qualquer parte interessada.

\section{Quadro 1: Contabilização de Derivativos}

\begin{tabular}{|l|l|l|l|}
\hline \multirow{2}{*}{ Cash Flow Hedge } & \multicolumn{3}{|c|}{ Contabilização Variação } \\
\cline { 2 - 4 } & Froteção & $\begin{array}{l}\text { OCl e posteriormente } \\
\text { Resultado }\end{array}$ & Rorção Não Efetiva \\
\hline Fair Value Hedge & Proteção & Resultado - mesmo período & Resultado \\
\hline Moeda Estrangeira & Proteção & Idem & Idem \\
\hline Hedge & Especulação & Imediatamente Resultado & \\
\hline
\end{tabular}

Fonte: hedge - US GAAP - SFAS 133

Apenas como esclarecimento adicional, os itens de hedge são os "objetos" alvo de proteção das firmas e os instrumentos de hedge referem-se aos direitos e obrigações estabelecidos por meio dos contratos financeiros de derivativos.

\subsection{Operacionalização e Efetividade}

Como já explicitado, o benefício de operações com derivativos advém do procedimento de a) compensar a exposição das mudanças no fair value do item de hedge; e b) proteger a exposição da variabilidade dos fluxos de caixa vinculados a operações 
de hedge, atribuível ao risco a ser mitigado ou eliminado. A documentação deve compreender justificativas e explicações razoáveis para o início da operação. Devido a essa dificuldade de implementação, as empresas incorrem em altos custos diretos e indiretos ao optarem, sendo estes últimos, referentes a consequências econômicas reais originadas do aumento da volatilidade do resultado e/ou patrimônio.

Baseado no conceito de que a não-efetividade de operações de hedge (falta de redução da exposição ao risco) se correlaciona com a especulação, Zhang (2006) conclui que existe evidência de que o SFAS 133 reduz o uso especulativo de derivativos; de qualquer forma, a correlação apontada é indireta, pois não é possível determinar por meio do uso de dados passados se as firmas usam derivativos para se proteger ou especular.

Conforme Pirchegger (2004), apesar da forma com que as regras de Hedge Accounting são implementadas e/ou executadas, sua aplicação revela-se uma boa opção, em vez de uma obrigação, para as firmas. E isso sugere que os responsáveis pela elaboração e pelo desenvolvimento/evolução das regras de contabilização de operações com derivativos esperam que os acionistas na figura de suas companhias se interessem em se adequar às normas de Hedge Accounting (não havendo obrigatoriedade). Justamente devido à sua complexidade, a sua melhor compreensão se faz essencial para uso corrente.

Conforme as normas contábeis, operações de hedge não são consideradas como de Hedge Accounting, quando as condições estipuladas no SFAS 133 não são satisfeitas ou quando as firmas simplesmente optam por não designar sua posição como Hedge Accounting ou quando elas têm a intenção de propiciar maior alavancagem por meio de especulação.

As variáveis que influenciam a contratação de operações de hedge podem ser de diversas naturezas, assim, nesta pesquisa restringir-se-á ao fator tamanho das empresas por meio, principalmente, de seu grau de faturamento e lucro bruto, e ao fator dívida, com o propósito de tentar demonstrar sua devida correlação. Outras variáveis também serão consideradas, como já mencionado, com a intenção de agregar valor à pesquisa.

\section{METODOLOGIA}

\subsection{Seleção da Amostra e Coleta de Dados}

Considerando apenas as empresas listadas na Bolsa de Valores de Nova lorque (NYSE - New York Stock Exchange) que atuam nos setores de Mineração, Siderurgia e Metalurgia e Papel e Celulose, foi estabelecida a amostra para o trabalho em questão. Houve a exclusão de empresas cujos ativos foram cancelados ou cujas informações se encontravam indisponíveis. Foram selecionadas 97 companhias através do banco de dados da Economatica, empresa especializada no fornecimento de informações cor- 
porativas, e também do website oficial da NYSE. Note-se que a intenção foi encontrar resultados consistentes que pudessem contribuir para a identificação de variáveis que exercem influência sobre a escolha contábil das firmas, no que se refere à aplicação da contabilidade de operações hedge (Hedge Accounting - SFAS 133), por meio de inferências.

A coleta de dados foi processada com o auxílio do mencionado banco de dados e também pela análise das demonstrações financeiras publicadas nos relatórios anuais, em dólares norte-americanos (US\$ milhões), de cada uma das selecionadas firmas, referentes ao ano de 2006, e listadas na NYSE, naquele mesmo período (parte dos dados não se encontrava tabulada em sistema). Os mencionados relatórios anuais, contendo as demonstrações financeiras das companhias, foram obtidos individualmente por meio de seus respectivos websites.

Foi possível identificar a existência de operações envolvendo derivativos, mais especificamente operações que alcançaram êxito, ou não, no chamado Teste de Efetividade e, por isso, foram contabilizadas como Hedge Accounting, ou não, respectivamente. Para efeito deste estudo, tal identificação se fez por meio da análise das Notas Explicativas contidas nos relatórios anuais do ano de 2006 de cada uma das empresas pesquisadas, uma vez que a evidenciação de operações de Hedge Accounting é exigida pelo SFAS 133. As firmas que contrataram operações de hedge e não mencionaram em seus respectivos relatórios anuais, no referido período, operações de Hedge Accounting, ou mencionaram não-efetividade, ou não relataram a efetividade de seus testes foram tratadas como se não possuíssem operações de Hedge Accounting; e as que evidenciaram, de alguma forma, o sucesso de seus testes de efetividade, foram classificadas como Hedge Accounting.

Foram selecionadas previamente variáveis independentes categóricas e nãocategóricas para formulação do modelo logit a ser explicitado. Os valores da variável dependente Hedge Accounting x Hedge foram estabelecidos como 0 ou 1, uma vez que o modelo a ser estabelecido é baseado no comportamento ou escolha assumida por empresas. Os dados foram tabulados e estruturados com base na equação de regressão mencionada abaixo.

$$
\begin{aligned}
& L_{i}=\operatorname{Ln}\left[P_{i} /\left(1-P_{i}\right)\right]=Y, \text { onde } P_{i} \text { representa a probabilidade }=> \\
& Y=\beta_{0}+\beta_{1} \cdot x_{1}+\beta_{2} \cdot x_{2}+\beta_{3} \cdot x_{3}+\beta_{4} \cdot x_{4}+\beta_{5} \cdot x_{5}+\beta_{6} \cdot x_{6}+\beta_{7} \cdot x_{7}+\beta_{8} \cdot x_{8}+\beta_{9} \cdot x_{9} \\
& +\beta_{10} \cdot x_{10}+\beta_{11} \cdot x_{11}+\beta_{12} \cdot x_{12}+\beta_{13} \cdot x_{13}+\varepsilon_{i}
\end{aligned}
$$

As variáveis são apresentadas de forma explicativa como demonstrado no Quadro 2 ilustrado abaixo. 


\section{Quadro 2: Discriminação Detalhada de Variáveis}

\begin{tabular}{|c|l|}
\hline Variável Dependente & \multicolumn{1}{c|}{ Proxy } \\
\hline & \\
$\mathrm{Y} \Rightarrow$ Empresa possui Hedge & $\begin{array}{l}\text { Análise de demonstrações financeiras das empresas selecionadas } \\
\text { Onde: }\end{array}$ \\
& $\begin{array}{l}\text { Accounting ou Hedge } \\
\text { - Empresa possui Hedge Accounting }=1\end{array}$ \\
& - Empresa possui Hedge (não possui Hedge Accounting) $=0$ \\
\hline
\end{tabular}

\begin{tabular}{|c|c|}
\hline Variável Independente * & Proxy \\
\hline $\mathrm{X}_{1} \Rightarrow>$ fatat & $=$ Faturamento / Ativo Total \\
\hline $\mathrm{X}_{2} \Rightarrow$ dvcpat & = Dívida de Curto Prazo / Ativo Total \\
\hline $\mathrm{X}_{3} \Rightarrow$ dvlpat & $=$ Dívida de Longo Prazo / Ativo Total \\
\hline $\mathrm{X}_{4} \Rightarrow>$ lbat & $=$ Lucro Bruto / Ativo Total \\
\hline $\mathrm{X}_{5} \Rightarrow \mathrm{ptb}$ & = Preço Ação Fechto. / Patrimônio Líquido (Price-to-Book - PTB) \\
\hline $\mathrm{X}_{6} \Rightarrow$ dummydv & $\begin{array}{l}\text { = Dummy Dívida Moeda Estrangeira ou Dólares Norte Americanos } \\
\text { Onde: } \\
\text { - Dívida Moeda Estrangeira }=1 \\
\text { - Dívida em Dólares Norte Americanos }(\text { US } \$)=0\end{array}$ \\
\hline$X_{7} \Rightarrow>$ dummyfat & $\begin{array}{l}\text { Dummy Faturamento Moeda Estrangeira ou Dólares Norte } \\
\text { Americanos } \\
\text { Onde: } \\
\text { - Faturamento Moeda Estrangeira }=1 \\
\text { - Faturamento em Dólares Norte Americanos }(\text { US } \$)=0\end{array}$ \\
\hline$X_{8} \Rightarrow$ dummypwc & $\begin{array}{l}=\text { Dummy PriceWaterHouseCoopers ou outra empresa de auditoria } \\
\text { Onde: } \\
\text { - Auditoria PriceWaterHouseCoopers }=1 \\
\text { - Outra empresa de auditoria }=0\end{array}$ \\
\hline$X_{9} \Rightarrow$ dummyey & $\begin{array}{l}=\text { Dummy Ernst \& Young ou outra empresa de auditoria } \\
\text { Onde: } \\
\text { - Auditoria Ernst \& Young }=1 \\
\text { - Outra empresa de auditoria }=0\end{array}$ \\
\hline $\mathrm{X}_{10}=>$ dummydelo & $\begin{array}{l}=\text { Dummy Deloitte \& Touche ou outra empresa de auditoria } \\
\text { Onde: } \\
\text { - Auditoria Deloitte \& Touche }=1 \\
\text { - Outra empresa de auditoria }=0\end{array}$ \\
\hline$X_{11}=>$ dummykpmg & $\begin{array}{l}\text { = Dummy KPMG ou outra empresa de auditoria } \\
\text { Onde: } \\
\text { - Auditoria KPMG }=1 \\
\text { - Outra empresa de auditoria }=0\end{array}$ \\
\hline $\mathrm{X}_{12}=>$ dummymine $\sim$ & $\begin{array}{l}=\text { Dummy Setor Mineração ou outro setor } \\
\text { Onde: } \\
\text { - Setor Mineração }=1 \\
\text { - Outra setor }=0\end{array}$ \\
\hline $\mathrm{X}_{13}=>$ dummyside $\sim$ & $\begin{array}{l}=\text { Dummy Setor Siderurgia/Metalurgia ou outro setor } \\
\text { Onde: } \\
\text { - Setor Siderurgia/Metalurgia }=1 \\
\text { - Outra setor }=0\end{array}$ \\
\hline
\end{tabular}

\subsection{Procedimentos}

As informações obtidas foram compiladas com o intuito de elaborar e analisar um modelo de regressão logit para dados não-agrupados ou individuais; adequando-se assim ao relato de Wooldridge (2006, p. 529):

Modelos de resposta binária aplicam-se com pequenas modificações a cortes transversais agrupados independentemente ou a outros conjuntos de dados nos quais as observações são independentes, mas não necessariamente identicamente distribuídas. [...]

Segundo Corrar, Paulo e Filho (2007, p. 284), a regressão logística se caracteriza como uma técnica estatística de classificação de fenômenos ou indivíduos em categorias 
específicas, além da estimação da probabilidade de ocorrência de determinado evento que possa se enquadrar nas mencionadas categorias, em face de um conjunto de variáveis explanatórias ou independentes.

Trata-se, sobretudo, de um modelo de probabilidade de resposta, no qual a variável dependente é qualitativa e dicotômica, cujo objetivo é a constatação da provável relação entre variáveis contábeis preestabelecidas e a opção pelo regime de Hedge Accounting efetuada pelas empresas, incluindo seus esforços em seguir as regras do FASB e obter sucesso na qualificação das operações de hedge. Resumidamente, trata-se de estimar as chances de se contratarem operações de derivativos com o intuito de enquadrá-las conforme o regime de Hedge Accounting estabelecido pelo FASB, ao se modificarem os valores das variáveis pré-selecionadas, as quais, algumas, por sua vez, têm relação direta com o tamanho das firmas e o nível de dívida (probabilidade condicional de que o evento ocorra).

Gujarati (2006, p. 481) expõe que a função de distribuição logística pode ser representada como segue abaixo, sendo $P_{i}$ a probabilidade, $X_{i}$ as variáveis explanatórias selecionadas e $\mathrm{Y}=1$ a representação de que a empresa possui operações de Hedge Accounting

$$
P_{i}=E\left(Y=1 / X_{i}\right)=1 /\left(1+e^{-\left(\beta_{1}+\beta_{2} X^{i}\right)}\right)
$$

Podendo ser escrita de outra forma para facilitar,

$$
P_{i}=1 /\left(1+e^{-z i}\right)=e^{z} /\left(1+e^{z}\right) ; \text { onde, } Z_{i}=\beta_{1}+X_{i .} \beta
$$

Wooldridge (2006, p. 519) expõe que o logit varia de $-\infty$ e $+\infty$. Além de $P_{i}$ ser nãolinear em $X$, é também não-linear nos $\beta$ - ver Equação 2. A mencionada equação pode ser linearizada, pois, se a probabilidade $P_{i}$ é dada pela Equação 3, então $\left(1-P_{i}\right)$, que trata da probabilidade de a empresa não aplicar contabilidade de operações de hedge (Hedge Accounting), é dado por:

$$
1-P_{i}=1 /\left(1+e^{-z i}\right)
$$

Assim,

$$
P_{i} /\left(1-P_{i}\right)=\left(1+e^{z i}\right) /\left(1+e^{-z i}\right)=e^{z i} ; \text { onde, } \operatorname{Pr}(\mathrm{Y}=1)=P_{i} ; \operatorname{e~} \operatorname{Pr}(\mathrm{Y}=0)=\left(1-P_{i}\right)
$$

$P_{i} /\left(1-P_{i}\right)$ nada mais é do que a razão da probabilidade ou das chances de uma firma possuir operações de Hedge Accounting contra a de não possuí-las.

Elaborando o logaritmo natural da equação (3), obtém-se linearidade em X e também nos $\beta$, resultando no modelo logit.

$$
L_{i}=\operatorname{Ln}\left(P_{i} / 1-P_{i}\right)=Z_{i}=>Z_{i}=\beta_{1}+\beta_{2} \cdot X_{i}
$$


Gujarati (2006, p. 482) afirma que enquanto o modelo de probabilidade linear pressupõe uma relação linear entre $\mathrm{Pi}$ e Xi , o modelo logit pressupõe que o logaritmo da razão de chances se relaciona linearmente com Xi. Corrar, Paulo e Filho (2007, p. 288) também relatam que, no modelo logit, a variação de probabilidade não é linear, e que existe um efeito marginal decrescente, isto é, quanto mais a probabilidade assumir valores próximos dos extremos 0 ou 1, espera-se que as mudanças ocorridas no coeficiente produzam efeitos cada vez menores sobre a variável dependente.

A função de máxima verossimilhança permite a evidenciação de mudanças nas interrelações dos logs da variável dependente e, não, na própria variável, como acontece com a linear, conforme Corrar, Paulo e Filho (2007, p. 284).

Assim, a interpretação do coeficiente de cada variável independente deve ser de que eles exercem efeitos sobre a quantidade de logaritmo natural da razão de chance (logit) e, não, diretamente sobre as probabilidades. É importante também ressaltar que eles indicam a tendência de aumento (coeficiente positivo) ou diminuição (coeficiente negativo) da mencionada probabilidade.

Considerando-se que as premissas de normalidade e independência de erros serão respeitadas, os resultados serão construídos a partir da ferramenta robust, que pode corrigir possíveis problemas estatísticos, devido à existência de heterocedasticidade.

Optou-se por elaborar um modelo de regressão logit, utilizando-se de todas as variáveis preestabelecidas, conforme mencionado abaixo, uma vez que testes com número de variáveis reduzido foram realizados e o resultado, de forma geral, não se alterou significativamente.

$Y=\beta_{0}+\beta_{1 . \text { fatatx }_{1}}+\beta_{2 .} \cdot$ dvcpatx $_{2}+\beta_{3 .} \cdot$ dvlpatx $_{3}+\beta_{4 . \text { lbatx }_{4}}+\beta_{5 . p t b x_{5}}+\beta$

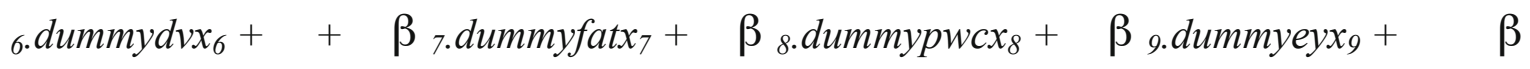

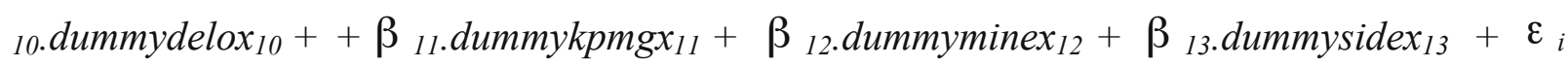

Onde $Y$ representa a escolha e o esforço das companhias selecionadas no que se refere à contratação e à qualificação de suas operações de hedge como Hedge Accounting; fatatx $_{1}$ é a razão entre faturamento e ativo total e está ligado ao tamanho da companhia; dvcpatx $_{2}$ indica a dívida de curto prazo da companhia; dvlpatx ${ }_{3}$ representa a dívida de longo prazo da firma; lbat $_{4}$ representa o lucro bruto e remete ao tamanho da empresa; ptbx5 é o índice price-to-book; dummydvx e dummyfatx $_{7}$ são variáveis dummy de dívida e faturamento, respectivamente, em moeda estrangeira, ligadas à contratação de operações com o intuito de proteção contra variação cambial, onde é igual a 1 se a maior parte $(>50 \%)$ da dívida e/ou faturamento da companhia é em moeda diferente do dólar norte americano, e zero, caso contrário; dummypwcx ${ }_{8}$, dummyeyx , dummydelox $_{10}$ e dummykpmgx ${ }_{11}$ são variáveis dummy relacionadas a empresas de auditoria big four, onde é igual a 1 se a em- 
presa de auditoria contratada é uma big four e igual a zero, caso contrário; dummyminex ${ }_{12}$ e dummysidex ${ }_{13}$ são variáveis dummy de controle ligadas ao setor da economia; e $\varepsilon_{i}$ é o termo de erro para as empresas no ano de 2006.

Espera-se constatar a existência de relação direta entre o comportamento das empresas no que se refere à escolha pela qualificação de operações de Hedge Accounting, e as variáveis explanatórias selecionadas, uma vez que estão ligadas a fatores que geram volatilidade no Resultado, como taxa de juros e variação cambial, e ao tamanho das empresas, conforme pesquisa de Saito e Schiozer (2005, p. 97-107). Espera-se também verificar a interação entre a mencionada escolha efetuada pelas empresas (variável dependente) e a presença de firmas de auditoria contratadas pelas companhias listadas na NYSE por meio da amostra selecionada.

\section{ANÁLISE DOS RESULTADOS}

Neste tópico são apresentados os resultados e a análise de regressão logit, para responder à questão de pesquisa: os fatores "tamanho" e "dívida" influenciam positivamente a escolha contábil de companhias para qualificação de operações como Hedge Accounting?

\section{Modelo Regressão Logit}

Observa-se que os coeficientes angulares parciais $(\beta)$ não medem a variação no logit estimado para qualquer variação unitária do valor dado através da variável independente, mantendo-se constante todas as demais variáveis. De forma mais clara, uma vez que a variável dependente só pode assumir dois valores (0 ou 1), não se pode interpretar $\beta$ como a variação prevista na probabilidade de sucesso quando a variável independente incorre em oscilação unitária (as probabilidades não são lineares em $X$ ). Assim, é necessário utilizar a regra prática de aproximação para escalonar os coeficientes do modelo Logit para obtenção dos efeitos sobre a probabilidade de resposta (média da função não-linear), onde multiplicam-se os mencionados coeficientes $\beta$ por 0,25 para torná-los comparáveis às estimativas do modelo de probabilidade linear e, dessa forma, proceder com a interpretação que permite a correlação direta entre coeficientes e variável dependente.

Como esclarecimento adicional, conforme Gujarati (2006, p. 486), a variação das probabilidades é a variação por unidade de alteração nas variáveis independentes. Para se calculá-la, à medida que o valor das variáveis independentes $\left(\mathrm{X}_{\mathrm{i}}\right)$ se alteram, é necessário observar que existe influência, tanto do coeficiente angular $(\beta)$, quanto do nível de probabilidade em relação ao qual a variação é medida; este último, por sua vez, depende do nível de $X_{i}$ no qual a probabilidade é medida. Resumidamente, a variação da probabilidade depende de e do nível de probabilidade, que, por sua vez, depende de $X_{i}$. A derivada da probabilidade em relação a $X_{i}$ é dada como:

$$
d P / d X=\beta \cdot P(1-P)
$$


Em complemento, considerando uma variável dependente binária, se o efeito de uma unidade de variação em $X_{i}$ sobre a probabilidade $(P)$ é máximo quando $P=0,5$ e mínimo quando $P$ está próximo de 0 ou 1 , a média das variáveis independentes $\left(X_{i}\right)$ é aproximadamente igual a 0,25.

\section{RESULTADOS}

Apenas como referência para avaliação da eficácia do modelo, o mesmo foi testado sem a inclusão de variáveis independentes e deixou-se guiar pela situação em que se enquadra a maioria dos casos observados, isto é, quando as empresas não qualificam suas operações como Hedge Accounting (ocorrência de 43 casos de firmas que possuem Hedge Accounting e 54 daquelas que não possuem tal qualificação). Dessa forma, o modelo classificaria incorretamente as companhias com Hedge Accounting ( $0 \%$ de acerto) e atingiria um percentual geral de acerto de $55,7 \%$. Ao se incluírem todas as variáveis explanatórias pré-selecionadas, o nível de acerto do modelo para a categoria de firmas com operações não-enquadradas como Hedge Accounting migraria de $100 \%$ para $72,2 \%$, porém, na média geral, sofreria um aumento $7,20 \%$, alterando-se de $55,7 \%$ para $62,9 \%$. Isso sugere que o modelo possui, em média, $62,9 \%$ de acurácia em suas predições, no que se refere às empresas com operações de Hedge Accounting ou não.

Deve-se identificar uma relação significativa que possa, estatisticamente, explicar a ocorrência de determinado evento. Assim, os testes de significância são importantes, pois permitem a verificação da acuracidade das predições desejadas. Com a intenção de testar a possibilidade de uso do modelo, isto é, testar o acerto na estimação de valores para a variável dependente em função dos valores das variáveis independentes, deve-se comprovar a refutação da hipótese de que todos os coeficientes da equação são nulos. Para tal, utilizou-se o modelo conhecido como Chi-Square, que gera um número referente à diferença entre o indicador Likelihood Value, obtido quando se inclui apenas a constante do modelo, e o gerado após a inclusão de todas as variáveis independentes.

O resultado do teste Qui-Quadrado (Model Chi-Square), que é semelhante ao teste F efetuado no modelo de regressão linear, de 13,210, permite concluir que pelo menos um dos coeficientes de regressão é diferente de zero (nem todos os parâmetros estimados são nulos), uma vez que, com a inclusão de variáveis independentes no modelo, espera-se que o Likelihood Value sofra uma redução estatisticamente significante. Assim, pode-se afirmar que tais variáveis contribuem para melhorar a qualidade das predições. Da mesma forma, os testes Step e Block têm o mesmo significado do anterior (Model Chi-Square) e resultados semelhantes, uma vez que se utilizou o método Enter, ou seja, incluíram-se simultaneamente todas as variáveis independentes. Outros indicadores considerados como Pseudo $\mathrm{R}^{2}$ também contribuem para a avaliação do desempenho geral do modelo, como é o caso do Cox \& Snell que indicou que cerca de $12,7 \%$ das variações ocorridas no log da razão de 
chance são explicadas pelo conjunto das variáveis independentes; e do Nangelkerke, que nada mais é do que a versão do indicador explicitado, anteriormente adaptado para fornecer resultados entre 0 e 1, e que, por sua vez, refletiu a possibilidade de o modelo ser capaz de explicar cerca de $17,0 \%$ das variações registradas na variável dependente.

Com o objetivo de testar a hipótese de que não há diferenças entre os resultados previstos pelo modelo e os observados, utilizou-se o Teste Hosmer e Lemeshow, que é um teste Qui-Quadrado e que apresentou a estatística de 10,245 e um nível de significância de 0,248 , indicando assim que os valores observados não são significativamente diferentes dos previstos, permitindo-se concluir, portanto, que há indícios de que o modelo pode ser utilizado para estimar a probabilidade de uma firma contratar operações de Hedge Accounting em função das variáveis independentes. Apenas como informação adicional, sendo o nível de significância encontrado $(0,248)$ superior a 0,05 , aceita-se a hipótese de que não existem diferenças significativas entre os valores observados e os previstos.

Com base na estatística Wald, que é um mecanismo semelhante à distribuição t de student, gerada pelo modelo e apresentada na Tabela 1, verifica-se que nenhum dos resultados gerados são nulos, refutando assim, a suposta hipótese nula de que poderiam ser iguais a zero, e reforçando a utilização das variáveis no modelo.

Tabela 1: Resultados Regressão Logit Hedge Accounting X Hedge

\begin{tabular}{|l|c|c|c|c|c|}
\hline $\begin{array}{l}\text { Variáveis } \\
\text { independentes }\end{array}$ & Sinais esperados & Coeficiente & P-Valor & Desvio padrão & Wald \\
\hline Fatatx1 & + & 0,060 & 0,916 & 0,571 & 0,011 \\
\hline Dvcpatx2 & + & 6,778 & 0,446 & 8,884 & 0,582 \\
\hline Dvlpatx3 & + & 3,183 & 0,048 & 1,612 & 3,898 \\
\hline Ibatx4 & + & 1,702 & 0,197 & 1,318 & 1,668 \\
\hline PTBx5 & - & 0,032 & 0,945 & 0,046 & 0,480 \\
\hline Dummydvx6 & + & 0,558 & 0,468 & 0,768 & 0,527 \\
\hline Dummyfatx7 & + & $-0,133$ & 0,844 & 0,676 & 0,039 \\
\hline Dummypwcx8 & - & $-2,326$ & 0,109 & 1,452 & 2,566 \\
\hline Dummyeyx9 & - & $-2,362$ & 0,096 & 1,418 & 2,776 \\
\hline Dummydelox10 & - & $-2,575$ & 0,084 & 1,491 & 2,983 \\
\hline Dummykpmgx11 & - & $-2,108$ & 0,156 & 1,485 & 2,016 \\
\hline Dummyminex12 & $?$ & $-0,722$ & 0,349 & 0,772 & 0,876 \\
\hline Dummysidex13 & $?$ & $-0,098$ & 0,900 & 0,779 & 0,016 \\
\hline
\end{tabular}

Esta tabela apresenta os resultados gerados pelo modelo de regressão logit definido com a intenção de investigar se os fatores determinantes pré-selecionados influenciam a escolha contábil de empresas listadas na NYSE nos setores de Mineração, Siderurgia e 
Metalurgia e Papel e Celulose, na qual a variável dependente é igual a 1 se as operações da empresa são qualificadas como Hedge Accounting e igual a zero, caso contrário. Esta tabela se refere ao modelo de regressão apresentado no texto por meio da equação 9; fata$\mathrm{tx}_{1}$ denota a razão entre faturamento e ativo total e está ligado ao tamanho da companhia; dvcpatx $_{2}$ indica o endividamento de curto prazo; dvlpatx ${ }_{3}$ representa o endividamento de

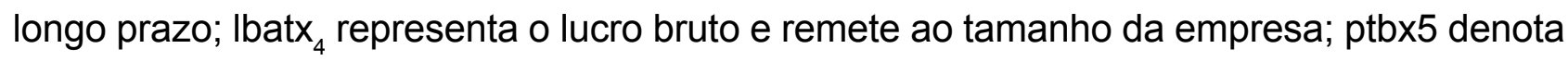
o índice price-to-book; dummydvx e dummyfatx $_{7}$ representam variáveis dummy de dívida e faturamento, respectivamente, em moeda estrangeira ligadas à contratação de operações com o intuito de proteção contra variação cambial, onde é igual a 1 se a maior parte (> 50\%) da dívida e/ou faturamento da companhia é em moeda diferente do dólar norte americano, e zero, caso contrário; dummypwcx ${ }_{8}$, dummyeyx , dummydelox $_{10}$ e dummykpmgx denotam $_{11}$ variáveis dummy relacionadas a empresas de auditoria big four, onde é igual a 1 se a empresa de auditoria contratada é uma big four e igual a zero, caso contrário; dummyminex ${ }_{12} \mathrm{e}$ dummysidex $_{13}$ são variáveis dummy ligadas ao setor da economia.

Os resultados apresentados na Tabela 1 demonstram que a escolha das empresas em contratar operações de hedge e qualificá-las como Hedge Accounting é positivamente relacionada com as variáveis de faturamento (fatatx ${ }_{1}$ ) e lucro bruto ( $\left(\operatorname{lbatx}_{4}\right)$, que, por sua vez, se relacionam com o tamanho das companhias, corroborando com os argumentos de Saito e Schiozer (2005) e Fenn, Post e Sharpe (1996) de que existe economia de escala para uso de derivativos; e também com as variáveis dívida de curto e longo prazo (dvcpatx ${ }_{2}$ e dvlpatx ${ }_{3}$, respectivamente), o que é sustentado por Saito e Schiozer (2005) e por DeMarzo e Duffie (1995), quando relatam que as taxas de juros são uma das principais fontes de volatilidade. De outra forma, verifica-se que tal escolha é negativamente relacionada com as quatro variáveis independentes dummy preestabelecidas referentes à presença de firmas de auditoria big four (dummypwcx ${ }_{8}$, dummyeyx , dummydelox $_{10}$ e dummykpmgx ${ }_{11}$ ), uma vez que tais firmas atribuem alto grau de exigência nos processos de avaliação para qualificação de operações como Hedge Accounting.

Considerando que $\mathrm{Y}$ é uma variável binária, um caminho adequado para a interpretação do modelo seria determinar a variação da probabilidade de um evento acontecer por meio da utilização da regra prática de aproximação, resultando, no caso da variável dvlpa$\mathrm{tx}_{3}$, em um valor de 0,7958 (coeficiente angular de 3,1830570 multiplicado por 0,25). Assim, a probabilidade de a empresa contratar operações a serem enquadradas como Hedge Accounting (altamente efetivas) seria de aproximadamente $79,58 \%$ maior se a dívida de longo prazo aumentasse na ordem de US\$1MM.

A Tabela 2 demonstra a suposta alteração de probabilidade referente à escolha contábil em questão, para cada oscilação de variável independente, na ordem de US\$1MM, considerando-se as demais variáveis constantes. É importante notar a significância estatística de cada variável. 
Tabela 2: Efeitos sobre s Probabilidade de Resposta

\begin{tabular}{|c|c|c|c|}
\hline $\begin{array}{l}\text { Variáveis } \\
\text { independentes }\end{array}$ & Coeficiente Angular & Wald & $\begin{array}{c}\text { Regras Práticas de } \\
\text { Aproximação Logit }(\mathrm{b} \times 0,25)\end{array}$ \\
\hline Fatatx & 0,060 & 0,011 & $2 \%$ \\
\hline Dvcpatx $_{2}$ & 6,778 & 0,582 & $1,69 \%$ \\
\hline Dvlpatx $_{3}$ & 3,183 & 3,898 & $80 \%$ \\
\hline Ibatx $_{4}$ & 1,702 & 1,668 & $43 \%$ \\
\hline $\mathrm{PTBx}_{5}$ & 0,032 & 0,480 & $0 \%$ \\
\hline Dummydvx & 0,558 & 0,527 & $14 \%$ \\
\hline Dummyfatx $_{7}$ & $-0,133$ & 0,039 & $-3 \%$ \\
\hline Dummypwcx $_{8}$ & $-2,326$ & 2,566 & $-58 \%$ \\
\hline Dummyeyx $_{9}$ & $-2,362$ & 2,776 & $-59 \%$ \\
\hline Dummydelox $_{10}$ & $-2,575$ & 2,983 & $-64 \%$ \\
\hline Dummykpmgx $_{11}$ & $-2,108$ & 2,016 & $-53 \%$ \\
\hline Dummyminex $_{12}$ & $-0,722$ & 0,876 & $-18 \%$ \\
\hline Dummysidex $_{13}$ & $-0,098$ & 0,016 & $-2 \%$ \\
\hline
\end{tabular}

Esta tabela apresenta, com base na regra de aproximação, a suposta alteração de probabilidade referente à escolha contábil (Hedge Accounting x Hedge) para cada oscilação de variável independente, na ordem de US\$1MM, considerando-se as demais variáveis constantes. ${ }^{*},{ }^{* *},{ }^{* * *}$ são estatisticamente significantes aos níveis de $1 \%, 5 \%$ e $10 \%$ respectivamente.

O coeficiente angular relacionado à variável Price-to-Book $\left(\mathrm{ptbx}_{5}\right)$ demonstra uma relação positiva com a variável dependente, não corroborando com o fato de que quanto maior (PTB), menor será o risco da empresa e a probabilidade de as companhias contratarem operações de derivativos e se qualificarem como Hedge Accounting.

Os valores dos coeficientes angulares gerados referentes às variáveis "dummydvx" $\mathrm{e}$ "dummyfatx" foram de 0,1394 e -0,1328 (regra de aproximação $=>\beta \times 0,25$ ), respectivamente, isto é, mais próximo de zero, assim, poder-se-ia interpretar que, apenas pelo fato de a dívida e o faturamento serem em moeda estrangeira, mantidas as outras variáveis constantes, não existem muitas chances de os esforços das firmas em obter Hedge Accounting serem influenciados.

Modelos de regressão com variável de escolha dicotômica, assumindo valores basicamente de 0 ou 1, como é o caso do selecionado (modelo logit), comumente utilizam a função de distribuição acumulada para sua representação. Outro ponto é que à medida que o tamanho da amostra aumenta, a distribuição tende à normalidade. Com isso, o software gera estatísticas Z para cada variável independente, e que, por sua vez, podem ser confrontadas com os valores da tabela de distribuição normal padronizada acumulada. Considerando que, para o estudo em questão, deve ser utilizado um teste de hipóteses bicaudal e "[...] visto que a distribuição é simétrica ao redor de $Z=0$, a área da cauda esquerda é igual à área da 
cauda direita correspondente" (GUJARATI, 2006, p. 776); utiliza-se um nível de significância ( ) de 0,05, cuja área em cada cauda da curva é de 0,4750, correspondendo ao Z crítico inferior e superior de 1,96 . Verifica-se que a variável independente que apresenta estatística $Z$ apurada fora da área de aceitação é a relacionada à dívida de longo prazo (dvlpatx $\left.{ }_{3}\right)$ e assim constata-se que se trata da única variável para a qual $\mathrm{H}_{0}$ é rejeitada. Conclui-se que, para a variável em questão, há indícios de que dívida de longo prazo pode exercer influência sobre a opção de uma empresa na contratação e na qualificação de suas operações de derivativos como Hedge Accounting (FASB), e que, por conseguinte, o porte da empresa também o pode, uma vez que este está ligado à capacidade da mesma de se endividar.

A Tabela 3 apresenta a estatística descritiva das variáveis da amostra em estudo. Verifica-se que as medianas das variáveis não-categóricas encontram-se próximas às suas respectivas médias, apesar de seus valores máximos se mostrarem distantes, tendendo para maior normalidade da distribuição.

\section{Tabela 3: Estatística Descritiva}

\begin{tabular}{|l|c|c|c|c|c|c|c|}
\hline $\begin{array}{l}\text { Variável dependente Hedge } \\
\text { accountingg } x \text { hedge }\end{array}$ & Média & Mediana & Mínimo & $\begin{array}{c}\text { Desvio } \\
\text { Padrão }\end{array}$ & Variância & N & Máximo \\
\hline & 0,443 & 0 & 0 & 0.499 & 0.249 & 97 & 1 \\
\hline Variáveis independentes & & & & & & & \\
\hline Fatatx & & & & & & \\
\hline Dvcpatx $_{2}$ & 0.817 & 0.810 & 0.075 & 0.492 & 0.242 & 97 & 2.607 \\
\hline Dvlpatx $_{3}$ & 0.022 & 0.010 & 0 & 0.028 & 0.001 & 97 & 0.150 \\
\hline Ibatx $_{4}$ & 0.188 & 0.161 & 0 & 0.147 & 0.022 & 97 & 0.825 \\
\hline PTBx $_{5}$ & 0.191 & 0.208 & -1.574 & 0.238 & 0.056 & 97 & 0.894 \\
\hline Dummydvx $_{6}$ & 4.077 & 2.953 & -19.509 & 5.998 & 35.979 & 97 & 33.967 \\
\hline Dummyfatx $_{7}$ & 0.175 & 0 & 0 & 0.382 & 0.146 & 97 & 1 \\
\hline Dummypwcx $_{8}$ & 0.299 & 0 & 0 & 0.460 & 0.212 & 97 & 1 \\
\hline Dummyeyx $_{9}$ & 0.412 & 0 & 0 & 0.495 & 0.245 & 97 & 1 \\
\hline Dummydelox $_{10}$ & 0.216 & 0 & 0 & 0.414 & 0.171 & 97 & 1 \\
\hline Dummykmmax $_{11}$ & 0.227 & 0 & 0 & 0.421 & 0.177 & 97 & 1 \\
\hline Dummyminex $_{12}$ & 0.124 & 0 & 0 & 0.331 & 0.110 & 97 & 1 \\
\hline Dummysidex $_{13}$ & 0.546 & 1 & 0 & 0.500 & 0.250 & 97 & 1 \\
\hline & 0.320 & 0 & 0 & 0.469 & 0.220 & 97 & 1 \\
\hline
\end{tabular}

Esta tabela apresenta a estatística descritiva dos dados pesquisados: fatatx ${ }_{1}$ denota a razão entre faturamento e ativo total e está ligado ao tamanho da companhia; dvcpatx ${ }_{2}$ indica o endividamento de curto prazo; dvlpatx ${ }_{3}$ representa o endividamento de longo prazo; Ibatx $_{4}$ representa o lucro bruto e remete ao tamanho da empresa; ptbx $_{5}$ denota o índice price-

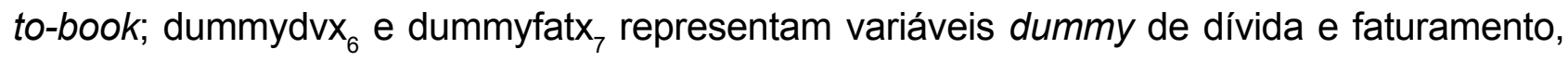
respectivamente, em moeda estrangeira ligadas à contratação de operações com o intuito de 
proteção contra variação cambial, onde é igual a 1 se a maior parte (> 50\%) da dívida e/ou faturamento da companhia é em moeda diferente do dólar norte americano, e zero, caso con-

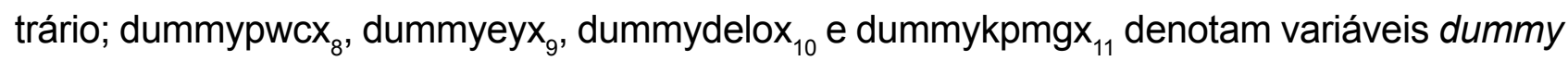
relacionadas a empresas de auditoria big four, onde é igual a 1 se a empresa de auditoria contratada é uma big four e igual a zero, caso contrário; dummyminex ${ }_{12}$ e dummysidex $_{13}$ são variáveis dummy ligadas ao setor da economia.

\section{CONCLUSÕES}

A pesquisa teve por objetivo analisar informações contábeis/financeiras de empresas dos setores de Mineração, Siderurgia e Metalurgia e Papel e Celulose listadas na bolsa de valores Nova lorque (NYSE), no ano de 2006, para melhor entendimento da sistemática da escolha contábil referente à contratação e a qualificação de operações de derivativos como Hedge Accounting, conforme as normas do FASB (SFAS 133), com o intuito de minimizar a volatilidade de seus resultados.

Com base em estudos realizados por autores cujos trabalhos foram utilizados nesta pesquisa, estabeleceram-se variáveis explicativas ligadas, principalmente, à dívida e ao tamanho das empresas, com o objetivo de verificar a existência de relação entre as mesmas e a escolha contábil em questão. Foram aplicados procedimentos estatísticos de análise de regressão logit, um a vez que a variável dependente é dicotômica e assume valores de 0 ou 1.

As evidências apontam para uma relação positiva e significante entre a dívida de longo prazo e a aplicação da contabilidade de operações de hedge (Hedge Accounting), o que é consistente com a teoria e corrobora com a relação esperada. Porém, não foram encontradas evidências significantes de que o tamanho das companhias, por meio das variáveis independentes pré-selecionadas, possui relação direta com sua escolha de contratação e qualificação de operações de derivativos como Hedge Accounting, conforme as normas do FASB (SFAS 133), apesar de outros estudos relatarem a existência de ganho de escala relacionado a tais operações. No que se refere a todas as outras variáveis, a hipótese nula foi aceita permitindo-se inferir que sua relação não pôde ser confirmada pelos resultados dos testes, uma vez que os mesmos não foram significantes estatisticamente.

Observou-se que todas as companhias pesquisadas, às quais possuíam operações envolvendo instrumentos de hedge, declararam que os utilizavam com a intenção específica de proteção/gerenciamento contra riscos e que o ponto que poderá determinar a escolha está ligado, principalmente, à natureza do item a ser protegido e/ou risco a ser mitigado.

Para futuros trabalhos, a possibilidade de expandir a amostra para outros setores e até mesmo a outros centros financeiros, além da NYSE, de forma a obter dados mais confiáveis e reais, é plausível. O tamanho da amostra teve grande influência nos resultados apresentados e pode ser considerado como um fator limitador da pesquisa em 
questão. A inclusão de outras variáveis contábeis e, principalmente, macroeconômicas (utilizando-se de outras variáveis dummy, por exemplo) podem auxiliar o pesquisador no caminho da maior abrangência e consequentemente na alta acuracidade e significância dos resultados. A influência da tributação nas decisões de gestores cujas firmas possuem ou possuirão operações envolvendo derivativos é outra linha que pode ser enfocada em pesquisas futuras.

Uma vez que os indícios de empresas brasileiras que optam pelo regime de Hedge Accounting ainda são escassos, e que tendem a aumentar devido à expansão da cultura de investimentos em ações e também ao viés de convergência para normas contábeis internacionais, este poderia ser considerado um bom momento para acompanhamento da evolução de tais normas, no que se refere às operações com derivativos, na visão das empresas brasileiras. Isto é, como se trata de uma transição é o momento ideal para estudar e discutir profundamente questões técnicas e práticas ligadas ao assunto, como, por exemplo, o possível efeito do arcabouço SFAS 133 nas companhias listadas na Bovespa.

\section{REFERÊNCIAS}

CARVALHO, Nelson M. Hedge Accounting de Ativos Financeiros Segundo o Pronunciamento SFAS 133: Análise dos Critérios de Reconhecimento de Receita em Face do Princípio da Realização da Receita e da Confrontação das Despesas. 2002. Dissertação (Mestrado em Controladoria e Contabilidade). Programa de Pós-Graduação em Controladoria e Contabilidade, Faculdade de Economia, Administração e Contabilidade da Universidade de São Paulo, São Paulo, 2002.

DEMARZO, Pter M.; DUFFIE, Darrel. Corporate Incentives for Hedging and Hedge Accounting. The Review of Financial Studies, vol. 8, no. 3, p. 743-771, 1995.

CORRAR, Luiz J.; PAULO, Edílson; DIAS FILHO, José Maria. Análise Multivariada: para Cursos de Administração, Ciências Contábeis e Economia. $1^{\text {a }}$ Ed., São Paulo: Atlas, 2007.

FASB - Financial Accounting Standards Board. SFAS 133 - Standard Accounting for Derivative Instruments and Hedging Activities. Disponível em: < http://www.fasb.org/st/summary/ stsum133.shtml >. Acesso em: 01 ago. 2007.

SFAS 137 - Accounting for Derivative Instruments and Hedging Activities - Deferral of the Effective Date of FASB Statement No. 133. Disponível em: < http://www.fasb.org/pdf/ fas137.pdf >. Acesso em: 06 jan. 2008. 
. SFAS 138 - Accounting for Certain Derivative Instruments and Certain Hedging Activities - An Amendment of FASB Statement No. 133. Disponível em: < http://www.fasb.org/ pdf/fas138.pdf >. Acesso em: 06 jan. 2008.

. SFAC no. 2 - Qualitative Characteristics of Accounting Information. Disponível em: < http://www.fasb.org/pdf/con2.pdf >. Acesso em: 06 jan. 2008.

FENN, George; POST, Mitch; SHARPE, Steven. Debt Maturity and the Use of Interest Rate Derivatives by Nonfinancial Firms. Capital Markets Section Federal Reserve Board paper, 1996.

GUJARATI, Damodar N. Econometria Básica. 4ª ed., Rio de Janeiro: Elsevier, 2006.

LIMA, Iran S.; LOPES, Alexsandro B. Perspectivas para a Pesquisa em Contabilidade: O Impacto dos Derivativos. Revista Contabilidade \& Finanças FIPECAFI - FEA. USP, São Paulo, v. 15, n. 26, p. 25, 2001.

LOPES, Alexsandro B.; Santos, Nelson S. A. Administração do Lucro Contábil e os critérios para Determinação da Eficácia do Hedge Accounting: Utilização da Correlação Simples dentro do Arcabouço do SFAS 133. Revista Contabilidade \& Finanças FIPECAFI - FEA. USP, São Paulo, n. 31, p. 16 - 25, 2003.

PIRCHEGGER, Bárbara. Hedge Accounting versus no Hedge Accounting for Cash Flow Hedges. Otto-von-Guericke-University, Magdeburg, 2004.

SAITO, Richard; SCHIOZER, Rafael. Uso de Derivativos em Empresas não-Financeiras Listadas em Bolsa no Brasil. RAUSP - Revista de Administração, São Paulo, 42, p. 97-107, 2007.

SMITH, Clifford W.; STULZ, René M. The Determinants of Firms' Hedging Policies. The Journal of Financial and Quantitative Analysis. University of Washington School of Business Administration, Vol. 20, No. 4, p. 391- 405, 1985.

WOOLDRIDGE, Jeffrey M. Introdução à econometria: uma abordagem moderna. São Paulo: Pioneira Thomson Learning, 2006.

ZHANG, Haiven. Effect of Derivative Accounting Rules on Corporate Risk-Management Behaviour. Ohio State University paper, 2006. 